\title{
Impacto da pandemia de COVID-19 na qualidade de vida e no estresse de docentes
}

\section{de uma instituição federal}

\author{
The impact of the COVID-19 pandemic on the quality of life and stress of teachers in a federal \\ institution \\ Impacto de la pandemia del COVID-19 en la calidad de vida y el estrés de los docentes de una \\ institución federal
}

ORCID: https://orcid.org/0000-0003-2467-9516 Instituto Federal de Educação, Ciência e Tecnologia do Paraná, Brasil E-mail: edivane.pedrolo@ifpr.edu.br

Leni de Lima Santana

ORCID: https://orcid.org/0000-0001-5480-7684 Instituto Federal de Educação, Ciência e Tecnologia do Paraná, Brasil E-mail: leni.santana@ifpr.edu.br

Nadine de Biagi Souza Ziesemer ORCID: https://orcid.org/0000-0002-5001-2347 Instituto Federal de Educação, Ciência e Tecnologia do Paraná, Brasil E-mail: nadine.biagi@ifpr.edu.br

Telma Pelaes de Carvalho ORCID: https://orcid.org/0000-0002-2912-02111 Instituto Federal de Educação, Ciência e Tecnologia do Paraná, Brasil E-mail: telma.carvalho@ifpr.edu.br

Tangriane Hainiski Ramos

ORCID: https://orcid.org/0000-0002-6641-9715 Instituto Federal de Educação, Ciência e Tecnologia do Paraná, Brasil E-mail: tangriane.ramos@ifpr.edu.br

Rafael Haeffner

ORCID: https://orcid.org/0000-0001-8848-6460 Escola de Saúde Pública de São José dos Pinhais, Brasil E-mail: rafaelhaeffner@gmail.com

\begin{abstract}
Resumo
A pandemia do SARS-CoV-2 declarada pela Organização Mundial da Saúde em 2020 impôs à população mudança de posturas e adaptação nos modos de vida em todos os contextos. Considerando que tais mudanças podem refletir negativamente na vida das pessoas, esta pesquisa tem como objetivo determinar o impacto da pandemia da COVID19 na qualidade de vida e no estresse de docentes do ensino básico, técnico e tecnológico de uma instituição federal de ensino. Trata-se de um estudo epidemiológico de corte transversal, cuja coleta de dados ocorreu de forma remota, nos meses de setembro a novembro de 2020 por meio da aplicação de um instrumento de identificação sociodemográfica, de formação e ocupacional, elaborada pelos autores, o WHOQOL-bref, versão brasileira e o Inventário de Sintomas de Estresse de Lipp. A obtenção dos resultados ocorreu por meio de análise descritiva e inferencial e evidenciou Índice Geral de Qualidade de Vida (IGQV) de 61,14\%. A situação conjugal, o fato de ter filhos, participar de comissões e possuir titulação máxima foi significativamente associado aos domínios físico, psicológico, das relações sociais e geral, respectivamente. Na avaliação de estresse 54,95\% tiveram avaliação positiva com predomínio na fase de resistência (90\%), com sintomatologia psicológica (66\%) e associação significativa em todos os domínios do WHOQOL-bref, demonstrando que o estresse interfere na qualidade de vida dos docentes. Desta forma, destaca-se a importância de se promover ações que objetivem a redução dos níveis de estresse para a melhoria na qualidade de vida dos docentes.
\end{abstract}

Palavras-chave: Qualidade de vida; Estresse psicológico; Isolamento social; Infecções por Coronavírus; Docentes; Ensino.

\begin{abstract}
The SARS-CoV-2 pandemic declared by the World Health Organization in 2020 imposed on the population a change in attitudes and adaptation in ways of life in all contexts. Considering that such changes may reflect negatively on people's lives, this research aims to determine the impact of the COVID-19 pandemic on the quality of life and stress of teachers in basic, technical and technological education at a federal educational institution. This is a cross-sectional epidemiological study, whose data collection occurred remotely, in the months of September to November 2020 through the application of an instrument of sociodemographic, training and occupational
\end{abstract}


identification, developed by the authors, the WHOQOL -bref, Brazilian version and Lipp's Stress Symptoms Inventory. The results were obtained through descriptive and inferential analysis and showed a 61.14\% General Quality of Life Index (IGQV). The marital situation, the fact of having children, participating in commissions and having a maximum degree was significantly associated with the physical, psychological, social relations and general domains, respectively. In the stress assessment, $54.95 \%$ had a positive assessment with predominance in the resistance phase $(90 \%)$, with psychological symptoms $(66 \%)$ and significant association in all domains of the WHOQOL-bref, demonstrating that stress interferes with quality of life of teachers. Thus, the importance of promoting actions that aim to reduce stress levels to improve the quality of life of teachers is highlighted.

Keywords: Quality of life; Psychological stress; Social isolation; Coronavirus infections; Faculty; Teaching.

\section{Resumen}

La pandemia de SARS-CoV-2 declarada por la Organización Mundial de la Salud en 2020 impuso a la población un cambio de actitudes y adaptación en los modos de vida en todos los contextos. Considerando que tales cambios pueden repercutir negativamente en la vida de las personas, esta investigación tiene como objetivo determinar el impacto de la pandemia COVID-19 en la calidad de vida y el estrés de los docentes de educación básica, técnica y tecnológica de una institución educativa federal. Se trata de un estudio epidemiológico transversal, cuya recolección de datos se realizó de forma remota, en los meses de septiembre a noviembre de 2020 mediante la aplicación de un instrumento de identificación sociodemográfica, formativa y ocupacional, el WHOQOL -bref, versión brasileña y Inventario de síntomas de estrés de Lipp. Los resultados se obtuvieron mediante análisis descriptivo e inferencial y mostraron un Índice General de Calidad de Vida (IGQV) del 61,14\%. La situación conyugal, el hecho de tener hijos, participar en comisiones y tener un grado máximo se asoció significativamente con los dominios físico, psicológico, relaciones sociales y general, respectivamente. En la valoración del estrés, el 54,95\% tuvo valoración positiva con predominio en la fase de resistencia (90\%), con síntomas psicológicos (66\%) y asociación significativa en todos los dominios del WHOQOL-bref, demostrando que el estrés interfiere en la calidad de vida de profesores. Así, se destaca la importancia de impulsar acciones que tengan como objetivo reducir los niveles de estrés para mejorar la calidad de vida de los docentes.

Palabras clave: Calidad de Vida; Estrés psicológico; Aislamiento social; Infecciones por Coronavirus; Docentes; Ensenãnza.

\section{Introdução}

O ano de 2020 foi globalmente marcado pelo impacto da pandemia do novo Coronavírus, SARS-CoV-2, na vida das pessoas, cujo epicentro teve início na cidade de Wuhan, na China, em 2019 e rapidamente se espalhou por todos os países (Rodrigues, Silva, Garcia Jr, Silva Jr, Gomes, Fernandes \& Oliveira. 2021; Souza et al., 2021). O SARS-CoV-2 é um vírus da família Coronaviridae com alto poder de virulência e patogenicidade e responsável pela ocorrência de uma série de agravos ao ser humano, entre elas a Síndrome Respiratória Aguda e afecções sistêmicas, condição conhecida como Corona Virus Disease (COVID), ou Doença do Coronavírus (Jiang, et al., 2020).

Frente à rápida disseminação, ao número de países afetados, ao crescente número de óbitos e a ausência de imunidade prévia na população, a Organização Mundial da Saúde (OMS) afirmou em janeiro de 2020 que o evento constituía uma Emergência de Saúde Pública de Importância Internacional (ESPII) e declarou estado de pandemia. (OMS, 2020)

O Brasil acompanhou a tendência mundial e em fevereiro de 2020, o Ministério da Saúde (MS) declarou situação de Emergência em Saúde Pública de Importância Nacional (ESPIN). Diante disso, foram divulgadas informações para o enfrentamento dos problemas de saúde pública a fim de antever os riscos da disseminação da doença causada pelo novo coronavírus em todo o território nacional (Brasil, 2020a).

Nesse contexto emergencial, medidas sanitárias foram recomendadas à população a fim de inibir a transmissão do SARS-CoV-2, tais como a intensificação da higienização das mãos, a adoção de etiqueta respiratória e do distanciamento social (Garcia \& Duarte, 2020)

No que tange ao distanciamento social, atendendo às recomendações de adoção de medidas de prevenção e contenção do avanço da infecção, o Ministério da Educação do Brasil (MEC), por meio da Portaria no 544 de 16 de junho de 2020 , autorizou em caráter excepcional, a substituição das disciplinas presenciais dos cursos regularmente autorizados, por atividades letivas que utilizassem recursos educacionais digitais, tecnologias de informação e comunicação ou outros meios 
convencionais enquanto durasse a situação de pandemia (Brasil, 2020b), de modo a evitar a aglomeração de pessoas nos espaços acadêmicos, bem como diminuir o número de pessoas circulando pela cidade.

Assim, diversas instituições educacionais suspenderam os calendários acadêmicos e administrativos, bem como eventos e outras atividades internas e externas, e conclamaram toda a comunidade a manter-se em distanciamento social. Frente a este cenário, foi necessário implementar novas modalidades de ensino visando a manutenção do processo ensinoaprendizagem dos estudantes (Avelino \& Mendes, 2020, Brasil, 2020b).

Paralelamente às adaptações curriculares e mudanças pedagógicas, outros desafios foram impostos aos docentes e estudantes. Distantes dos espaços acadêmicos, precisaram adequar ambientes de trabalho e estudos, aprender a utilizar novas tecnologias, dividir o tempo entre as demandas acadêmicas e familiares, além de conviver com o medo de ser infectado pelo SARS-CoV-2 (Ornell, Schuch, Sordi, \& Kessler, 2020; Souza, Jardim, Marques, Lopes Jr, Santos \& Liberato, 2021).

Adicionalmente a essas transformações, a dificuldade de acesso tecnológico e de domínio das tecnologias constituíram novos desafios para o processo de ensino-aprendizagem. O ensino remoto, além de despender mais tempo para a elaboração das atividades, gera custos para a aquisição de pacotes de acesso à internet, requer disponibilidade de tempo, “disciplina, compromisso, motivação, criatividade e vontade para sua implementação" (Nhantumbo, 2020), o que nem sempre é possível no contexto atual.

Diante desse cenário, tanto docentes como estudantes tiveram as suas rotinas alteradas, precisando ajustar-se a um novo modelo de ensino-aprendizagem e concomitantemente precisaram realizar ajustes necessários no âmbito familiar, trabalhista e social, para atenuar a repercussão negativa na saúde e na qualidade de vida de um modo geral (Rodrigues, Silva, Garcia Jr, Silva Jr, Dias \& Carvalho, 2021).

Pesquisas realizadas em outras pandemias evidenciaram que são inúmeros os impactos na qualidade de vida (QV) das pessoas quando se adotam medidas de distanciamento social. O medo e o estresse podem ser vividos pessoas saudáveis, e se intensificam em pessoas com transtornos psiquiátricos pré-existentes, o que pode interferir na qualidade de vida das pessoas, seja na esfera física, emocional, financeira e social (Lima, 2020; Ornell, Schuch, Sordi \& Sordi, 2020).

Dessa forma, pesquisas que investigam a temática da QV e o estresse na população são necessárias, uma vez que abordam diferentes aspectos da existência dos indivíduos os quais influenciam diretamente na forma como percebem a própria QV uma vez que se trata de uma avaliação subjetiva e intrinsecamente relacionada a fatores culturais, educacionais, morais e de hábitos de vida (Pinto, Dias, Salera, Silva \& Marinho, 2021). Assim, a QV é alterada pela condição de saúde, de moradia, de empregabilidade, de acesso à alimentação e ao saneamento básico, dentre outros fatores.

Frente ao exposto indaga-se: “As mudanças individuais e/ou coletivas advindas das ações adotadas para contenção do número de casos de COVID-19 interferiram na qualidade de vida e no estresse dos docentes do ensino básico, técnico e tecnológico?" Assim, a pesquisa tem como objetivo geral determinar o impacto da pandemia da COVID-19 na qualidade de vida e no estresse de docentes do ensino básico, técnico e tecnológico. E como objetivos específicos identificar os fatores que interferem na qualidade de vida dos docentes; verificar o nível de estresse dos docentes; e verificar a associação entre QV e estresse dos docentes durante a pandemia.

\section{Metodologia}

Trata-se de um estudo epidemiológico de corte transversal, um delineamento útil para descrever a situação do fenômeno estudado, pois possibilita ao pesquisador medir a prevalência de determinadas condições ou agravos à saúde da população de forma a subsidiar ações de intervenção (Hulley et al., 2015).

Foi realizado em uma instituição federal de ensino da região sul do Brasil, no período de setembro a novembro de 2020. A amostra foi de conveniência, composta por docentes das diferentes áreas do conhecimento que atuam no ensino 
básico, técnico e tecnológico da rede federal.

A coleta de dados ocorreu de forma remota, com auxílio da ferramenta Google Forms ${ }^{\circledR}$. Os docentes foram convidados a participar da pesquisa por meio de seus e-mails institucionais, disponibilizados pela direção de ensino. Após consentirem sua participação na pesquisa, mediante concordância com o Termo de Consentimento Livre e Esclarecido (TCLE), os docentes tinham permissão para acessar os instrumentos de coleta de dados, sendo eles, um instrumento de identificação sociodemográfica, de formação e ocupacional, elaborada pelos autores, o WHOQOL-bref, versão brasileira (Fleck et al., 2000) e o Inventário de Sintomas de Estresse de Lipp (ISSL) (Lipp, 2015).

O instrumento de identificação sociodemográfica, de formação e ocupacional continha 13 questões objetivas que indagavam sobre idade, sexo, raça ou etnia, deficiência física ou intelectual, situação conjugal, filhos, área de formação, titulação máxima, nível de atuação, regime de trabalho, se tem Dedicação Exclusiva, rotina pedagógica, participação em comissões, comitês ou conselhos na instituição e carga horária semanal destinada a participação nas mesmas.

O WHOQOL-bref foi aplicado para avaliar a qualidade de vida dos participantes no contexto estudado. Trata-se de um questionário de domínio público, estruturado e validado para a população brasileira e contém 26 questões distribuídas da seguinte forma: duas gerais (qualidade de vida geral e satisfação com a saúde) e 24 abrangendo os domínios: Físico, Psicológico, das Relações Sociais e do Meio Ambiente (Who, 1994).

A aplicação do Inventário de Sintomas de Estresse de Lipp (ISSL) foi utilizada para avaliar a ocorrência de estresse entre os docentes no contexto da pandemia. Trata-se de um instrumento elaborado e validado para indivíduos acima de 15 anos (Lipp, 2015), e sua licença para uso foi adquirida pelas pesquisadoras. É um instrumento composto por uma lista de sintomas físicos e psicológicos separados em três quadros referentes às fases do estresse (alerta, resistência/quase-exaustão e exaustão). O primeiro e o segundo trazem ambos 15 itens e o terceiro apresenta 22 itens, totalizando 52 itens avaliados.

Os critérios de inclusão foram: ser docente do ensino básico, técnico e tecnológico lotado no campus Curitiba. Foram excluídos da pesquisa docentes que estavam em afastamento do trabalho por período superior a seis meses, por não terem vivenciado a experiência de suspensão do calendário acadêmico e os que não preencheram mais de $20 \%$ das questões do questionário WHOQOL-bref. Foi realizado cálculo amostral, que determinou um mínimo de 62 docentes.

A análise dos dados foi realizada por profissional epidemiologista, um dos autores do estudo, e envolveu análise descritiva e inferencial. Para as variáveis contínuas foram feitas as medidas de tendência central (média e mediana) e de dispersão (desvio padrão, percentis e amplitude interquartil) e para as variáveis categóricas, frequências absolutas e relativas. Para variável modalidade de ensino onde atua foram considerados: curso técnico integrado ao ensino médio (técnico integrado), curso técnico subsequente ao ensino médio (técnico subsequente), graduação ou tecnólogo (graduação) e pósgraduação.

Para a análise dos dados decorrentes da aplicação do WHOQOL-bref, as duas questões gerais sobre QV foram calculadas em conjunto para gerar um único escore independente dos outros escores dos domínios, o qual denomina-se de Índice Geral de Qualidade de Vida (IGQV). Para os demais dados utilizou-se uma ferramenta construída por Pedroso, Pilatti \& Reis (2009), que realiza cálculos dos escores e a estatística descritiva de forma automatizada. A ferramenta utiliza a média aritmética simples dos escores das 26 questões do questionário - considerados os domínios Físico, Psicológico, das Relações sociais e do Meio ambiente - e as converte em uma escala de 0 a 100. O escore de cada domínio é obtido em uma escala positiva que indica que quanto mais alto o escore, melhor a qualidade de vida naquele domínio.

Já na análise do Inventário de Sintomas de Stress para Adultos (ISSL) foram utilizados os métodos padronizados elaborados pelos autores do instrumento e disponibilizados em um manual de aplicação e análise. A ocorrência de estresse é determinada se os escores brutos atingirem os limites determinados. A fase do estresse é determinada por porcentagens préestabelecidas, de acordo com os valores brutos dos sintomas. A maior porcentagem obtida indica a fase do estresse em que a 
pessoa se encontra. Caso haja empate entre as porcentagens, o diagnóstico é feito pela fase mais avançada (Lipp, 2015).

Para a caracterização da sintomatologia predominante, física ou psicológica, considera-se apenas a fase em que o indivíduo se encontra e compara-se às porcentagens obtidas nos sintomas físicos com as dos sintomas psicológicos, a que obtiver a maior porcentagem revela a sintomatologia predominante, conforme determina o manual (Lipp, 2015).

Para a etapa inferencial foram observadas as distribuições das proporções das categorias em cada variável e adotado o teste Qui-quadrado de Pearson ou Exato de Fisher, conforme adequado. Foi considerado significativo valor de $\mathrm{p}<0,05$. Para análise inferencial, optou-se por excluir os "Não informado" em cada uma das variáveis sociodemográficas, de formação e laborativas. Quanto à qualidade de vida, considerou-se que indivíduos com escore superior a 50\% possuíam qualidade de vida e menor ou igual a 50\% como não tendo qualidade de vida. As variáveis de desfecho foram: qualidade de vida e estresse.

A pesquisa foi aprovada pelo Comitê de Ética em Pesquisa da instituição sob CAAE 37024020.6.0000.8156. Foi respeitada a Resolução 466/2012, do Conselho Nacional de Saúde, para condução desta pesquisa.

\section{Resultados}

Foram incluídos 91 docentes na pesquisa. A idade média foi de 44,62 $\pm 8,40$ anos. No tocante à raça, houve 14 indivíduos que se autodeclararam não brancos, sendo 13 (14,29\%) pardos e um amarelo (1,10\%). Quanto à deficiência, os participantes afirmaram possuir deficiência: visual $(3,30 \%, \mathrm{n}=3)$, auditiva ou intelectual $(1,10 \%, \mathrm{n}=1 \mathrm{cada})$. Dos participantes que afirmaram ter filhos, 31,87\% $(n=29)$ têm um filho, 29,67\% $(n=27)$ dois filhos e 14,29\% ( $n=13)$ três ou mais filhos. Quanto à participação em comissões institucionais, a carga horária semanal informada pelos docentes foi, em média, de 8,94 \pm 8,09 horas, com mínimo de duas horas e máximo de 33 horas semanais. A Tabela 1 apresenta os demais dados sociodemográficos, de formação e relacionados ao trabalho dos participantes.

Tabela 1 - Características sociodemográficas, de formação e relacionadas ao trabalho dos docentes e análise inferencial de sua relação com a qualidade de vida geral. Brasil, 2021.

\begin{tabular}{|c|c|c|c|c|}
\hline \multirow[t]{3}{*}{ Variável } & \multirow[t]{2}{*}{ Descritivo } & \multicolumn{3}{|c|}{ Inferencial - Qualidade de vida } \\
\hline & & Sim & Não & P-valor \\
\hline & n (\%) & n (\%) & n (\%) & \\
\hline Sexo & & & & $0,680 *$ \\
\hline Masculino & $47(51,65)$ & $22(46,81)$ & $25(53,19)$ & \\
\hline Feminino & $43(47,25)$ & $22(48,89)$ & $21(48,84)$ & \\
\hline Não informado & $1(1,10)$ & & & \\
\hline Idade & & & & $0,231 *$ \\
\hline $1^{\circ}$ tercil (26 a 41 anos) & $34(37,36)$ & $19(55,88)$ & $15(44,12)$ & \\
\hline $2^{\circ}$ tercil ( 42 a 45 anos) & $27(29,67)$ & $15(55,56)$ & $12(44,44)$ & \\
\hline $3^{\circ}$ tercil (46 a 72 anos) & $30(32,97)$ & $11(36,67)$ & $19(63,33)$ & \\
\hline Raça & & & & $1,000 *$ \\
\hline Branco & $74(81,32)$ & $37(50,00)$ & $37(50,00)$ & \\
\hline Não branco & $14(15,39)$ & $7(50,00)$ & $7(50,00)$ & \\
\hline Não informado & $3(3,30)$ & & & \\
\hline Deficiência & & & & $0,203^{\neq}$ \\
\hline Sim & $5(5,50)$ & $4(80,00)$ & $1(20,00)$ & \\
\hline
\end{tabular}


Não

Não informado

\section{Situação conjugal}

Com companheiro

Sem companheiro

Não informado

\section{Filhos}

Sim

Não

Não informado

\section{Área de formação}

Ciência exatas e da terra ou engenharias

Ciências da saúde ou biológicas

Ciências humanas ou sociais aplicadas

Outras áreas

\section{Titulação máxima}

Especialização

Mestrado

Doutorado

Não informado

\section{Nível de ensino onde atua}

Técnico integrado

Técnico subsequente/graduação ou tecnólogo

Pós-graduação

Não informado

\section{Regime de trabalho}

Professor substituto 20h/40h

Professor efetivo 20/40h

Professor efetivo $40 \mathrm{~h}$ com dedicação exclusiva

\section{Rotina de atividades de ensino semanais}

Ministra uma grande carga horária

Ministra uma pequena carga horária

Não ministra atividades de ensino

Não informado

\section{Atuação em comitês}

Sim

Não

Não informado

\begin{tabular}{lll}
\hline $84(92,30)$ & $40(47,62)$ & $44(52,38)$ \\
$2(2,20)$ & & \\
\hline & & \\
$74(81,32)$ & $39(52,70)$ & $35(47,30)$ \\
$16(17,58)$ & $6(37,50)$ & $10(62,50)$ \\
$1(1,10)$ & &
\end{tabular}

$69(75,82) \quad 36(52,17) \quad 33(47,83)$

$21(23,08) \quad 8(38,10) \quad 13(61,90)$

$1(1,10)$

$22(25,29) \quad 10(45,45) \quad 12(54,55)$

$17(19,54) \quad 9(52,94) \quad 8(47,06)$

$27(31,03) \quad 16(59,26) \quad 11(40,74)$

$21(24,14) \quad 10(47,62) \quad 11(52,38)$

$\mathbf{0 , 0 0 1}{ }^{\neq}$

$5(5,49) \quad 1(20,00) \quad 4(80,00)$

$37(40,66) \quad 11(29,73) \quad 26(70,27)$

$48(52,74) \quad 32(66,67) \quad 16(33,33)$

$1(1,10)$

$41(45,05) \quad 19(57,58) \quad 14(42,42)$

$45(49,45) \quad 21(53,85) \quad 18(46,15)$

$4(4,40) \quad 3(75,00) \quad 1(25,00)$

$1(1,10)$

$5(5,50) \quad 2(40,00) \quad 3(60,00)$

$5(5,50) \quad 0(0,00) \quad 1(100,00)$

$81(89,00) \quad 43(50,59) \quad 42(49,41)$

\begin{tabular}{llll}
\hline & & & $0,615^{*}$ \\
$48(52,74)$ & $24(50,00)$ & $24(50,00)$ & \\
$35(38,46)$ & $16(45,71)$ & $19(54,29)$ & \\
$4(4,40)$ & $1(25,00)$ & $3(75,00)$ & \\
$4(4,40)$ & & & \\
\hline & & & \\
$51(56,04)$ & $29(58,00)$ & $21(42,00)$ & \\
$39(42,86)$ & $16(39,02)$ & $25(60,98)$ & \\
$1(1,10)$ & & &
\end{tabular}

Legenda: *Qui-quadrado de Pearson; ${ }^{\ddagger}$ Teste exato de Fisher. Fonte: Autores. 
Observa-se que a maioria dos participantes era do sexo masculino, com idade entre 26 e 41 anos, brancos, sem deficiência, casados/com companheiro e com filhos. Quanto à formação, as áreas com maior quantitativo de docentes foram as Ciências humanas e sociais aplicadas e a maioria dos docentes possui titulação de doutor. No tocante à atuação profissional, há uma predominância de docentes que atuam no ensino técnico subsequente ou na graduação/tecnólogo, em regime de dedicação exclusiva para a instituição, que ministra uma grande carga horária de atividades de ensino e, de forma complementar, participa de comissões institucionais. De todas as variáveis analisadas na Tabela 1, somente a titulação afetou de forma significativa a qualidade de vida dos docentes. Contudo, destaca-se que na variável "atuação em comitês", 15\% dos docentes com carga horária adicional nesta atividade possuem menor qualidade de vida, apesar do valor de p não ser significativo.

Os participantes possuem, em sua maioria, formação em uma única área de atuação $(94,51 \%, \mathrm{n}=86)$, sendo assim distribuídos: Ciências sociais aplicadas $(16,48 \%, \mathrm{n}=15)$; Ciências da saúde $(14,29 \%, \mathrm{n}=13)$; Ciências exatas e da terra, Ciência humanas e Linguística, letras e artes (13,19\%, n = 12 cada); Engenharias (10,99\%, n = 10); Ciências biológicas $(4,40 \%, \mathrm{n}=4)$; Ciências agrárias $(3,30 \%, \mathrm{n}=3)$; Outras áreas $(5,49 \%, \mathrm{n}=5)$; mais de uma área $(5,49 \%, \mathrm{n}=5)$.

Quanto à rotina de atividades escolares, a maioria dos docentes ministra grande carga horária de atividades remotas de ensino $(52,74 \%, \mathrm{n}=48)$, sendo que 36,26\% $(\mathrm{n}=33)$ afirmam não se sentirem sobrecarregados, embora 16,48\% $(\mathrm{n}=15)$ afirmem sobrecarga de trabalho. Dos docentes que ministram pequena carga horária de atividades remotas de ensino $(38,46 \%, \mathrm{n}=35)$, 23,08\% ( $\mathrm{n}=21$ ) ministram conteúdos que serão computados como carga horária das disciplinas dos cursos, enquanto 15,38\% ( $n=14)$ ministram somente atividades complementares de ensino, que não serão computadas como carga horária em nenhuma disciplina dos cursos. O nível prioritário de atuação dos docentes é o técnico subsequente ao ensino médio/graduação ou tecnólogo, sendo 30,77\% ( $\mathrm{n}=28$ ) no técnico subsequente e 18,68\% ( $\mathrm{n}=17$ ) na graduação ou tecnólogo.

No tocante à qualidade de vida relatada pelos docentes, o Índice Geral de Qualidade de Vida (IGQV) dos participantes foi de 61,14\%. Quanto aos domínios, o do "Meio ambiente" teve melhor avaliação (66,37\%), seguido das "Relações sociais" $(61,54 \%)$ e do domínio "Psicológico" (60,38\%), enquanto o "Físico" foi o que obteve menor pontuação $(54,17 \%)$.

Com relação às facetas do WHOQOL-bref, as que tiveram melhores avaliações foram: mobilidade $(86,81 \%)$, autoestima $(79,44 \%)$, transporte $(79,40 \%)$ e ambiente do lar $(77,20 \%)$. Já as facetas com as menores avaliações foram: dependência de medicamentos ou de tratamentos $(32,87 \%)$, dor e desconforto $(33,43 \%)$, pensamentos negativos $(36,39 \%)$ e recreação e lazer $(43,96 \%)$.

Na Tabela 2 são apresentadas as variáveis sociodemográficas, de formação e laborativas que tiveram significância estatística em cada um dos domínios do WHOQOL-bref. A situação conjugal teve interferência na avaliação do domínio "Físico", possuir filhos no domínio "Psicológico", atuar em comitês institucionais nos domínios das "Relações sociais" e do "Meio ambiente". Já na avaliação geral da qualidade de vida, somente a titulação mostrou interferir significativamente. Apesar de não apresentar relação estatisticamente significativa, a situação conjugal impactou no domínio das "Relações sociais", pois dos docentes que afirmaram ter boa QV, 49 (66,22\%) eram casados ou tinham companheiro, contra sete participantes solteiros que relataram QV boa $(\mathrm{p}=0,093)$. 
Tabela 2 - Variáveis sociodemográficas, de formação e laborativas com relação significativa com a qualidade de vida em cada um dos domínios do WHOQOL-bref. Brasil, 2021.

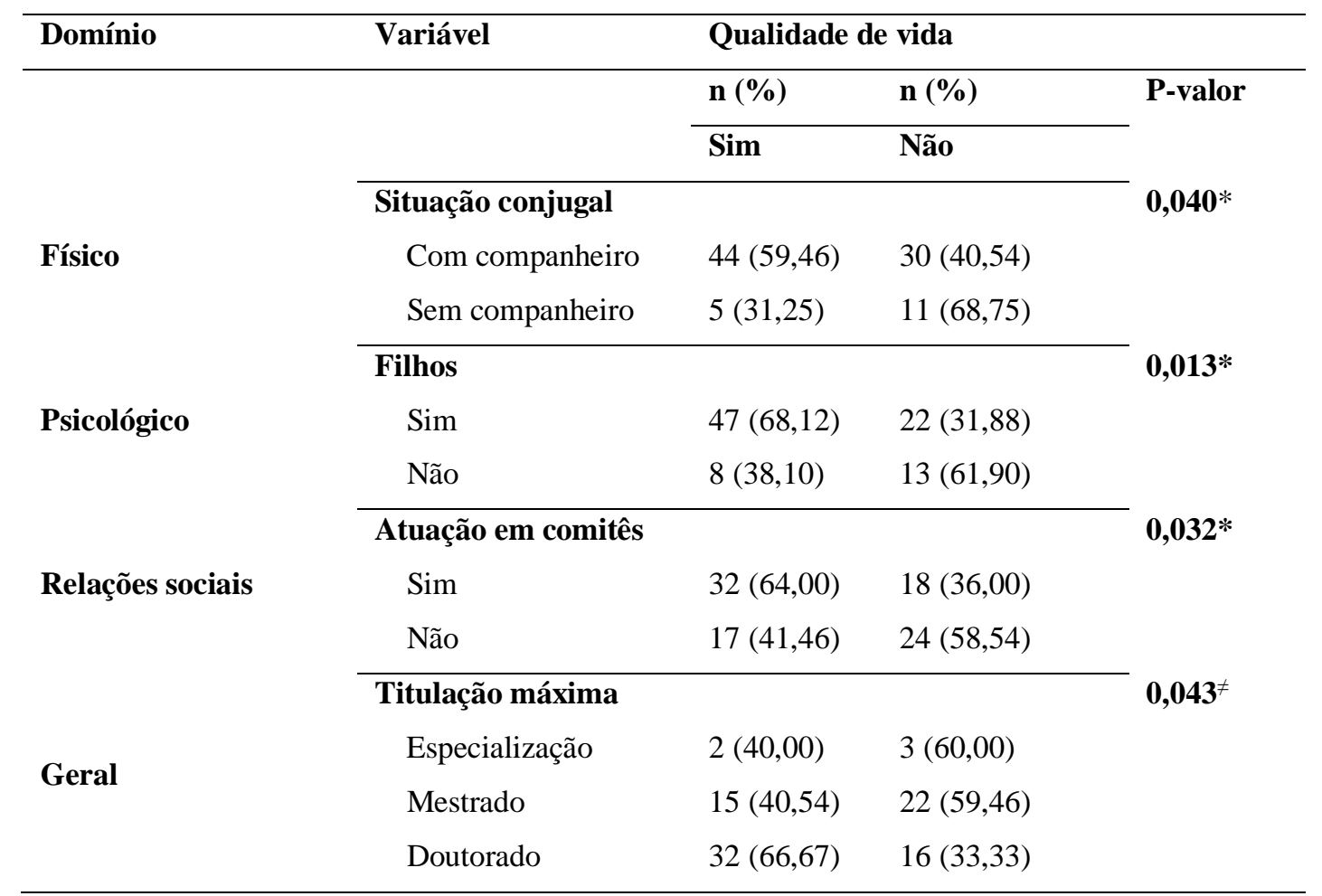

Legenda: *Qui-quadrado de Pearson; ${ }^{\neq}$Teste exato de Fisher.

Fonte: Autores.

Na Tabela 3 são apresentados os dados em relação ao nível de estresse e sintomatologia principal relatados pelos participantes. Houve predomínio de docentes com estresse em fase de resistência e em sua maioria com sintomatologia psicológica. Nenhum docente está na fase de alerta para o estresse.

Tabela 3 - Nível de estresse e sintomatologia principal apresentada pelos docentes. Brasil, 2021.

\begin{tabular}{lll}
\hline Variável & n & $\%$ \\
\hline Estresse & 50 & 54,95 \\
Sim & 41 & 45,05 \\
Não & & \\
Nível de estresse & 45 & 90,00 \\
$\quad$ Fase de resistência & 5 & 10,00 \\
Fase de quase-exaustão & & \\
Sintomatologia principal & 15 & 30,00 \\
Física & 33 & 66,00 \\
Psicológica & 2 & 4,00 \\
Ambos & & \\
\hline
\end{tabular}

Fonte: Autores.

A Tabela 4 apresenta os dados da análise inferencial do estresse dos docentes em relação às variáveis 
sociodemográficas, de formação e relacionadas ao trabalho. Nenhuma variável interferiu de forma significativa no estresse dos docentes, contudo observa-se um maior quantitativo de homens com estresse.

Tabela 4 - Análise inferencial do estresse dos docentes, segundo características sociodemográficas, de formação e relacionadas ao trabalho. Brasil, 2021.

\begin{tabular}{|c|c|c|c|}
\hline Variável & \multicolumn{3}{|l|}{ Estresse } \\
\hline & Sim & Não & P-valor \\
\hline & n $(\%)$ & n $(\%)$ & \\
\hline Sexo & & & $0,081 *$ \\
\hline Masculino & $28(65,12)$ & $15(34,88)$ & \\
\hline Feminino & $22(46,81)$ & $25(53,19)$ & \\
\hline Idade & & & $0,479 *$ \\
\hline $1^{\circ}$ tercil ( 26 a 41 anos) & $21(61,76)$ & $13(38,24)$ & \\
\hline $2^{\circ}$ tercil ( 42 a 45 anos) & $15(55,56)$ & $12(44,44)$ & \\
\hline $3^{\circ}$ tercil (46 a 72 anos) & $14(46,67)$ & $16(53,33$ & \\
\hline Raça & & & $0,710^{*}$ \\
\hline Branco & $41(55,41)$ & $33(44,59)$ & \\
\hline Não branco & $7(50,00)$ & $7(50,00)$ & \\
\hline Deficiência & & & $0,650^{\neq}$ \\
\hline Sim & $2(40,00)$ & $3(60,00)$ & \\
\hline Não & $48(57,14)$ & $36(42,86)$ & \\
\hline Situação conjugal & & & $0,476^{*}$ \\
\hline Com companheiro & $39(52,70)$ & $35(47,30)$ & \\
\hline Sem companheiro & $10(62,50)$ & $6(37,50)$ & \\
\hline Filhos & & & $0,738^{*}$ \\
\hline Sim & $39(56,52)$ & $30(43,48)$ & \\
\hline Não & $11(52,38)$ & $10(47,62)$ & \\
\hline Área de formação & & & $0,690 *$ \\
\hline Ciência exatas e da terra ou engenharias & $13(59,09)$ & $9(40,91)$ & \\
\hline Ciências da saúde ou biológicas & $10(58,82)$ & $7(41,18)$ & \\
\hline Ciências humanas ou sociais aplicadas & $12(44,44)$ & $15(55,56)$ & \\
\hline Outras áreas & $12(57,14)$ & $9(42,86)$ & \\
\hline
\end{tabular}




\section{Titulação máxima}

Especialização

Mestrado

Doutorado

\section{Nível de ensino onde atua}

Técnico integrado

Técnico subsequente/graduação ou tecnólogo

Pós-graduação

\section{Regime de trabalho}

Professor substituto 20h/40h

Professor efetivo 20/40h

Professor efetivo 40h com dedicação exclusiva

\section{Rotina de atividades de ensino semanais}

Ministra uma grande carga horária

Ministra uma pequena carga horária

Não ministra atividades de ensino

\section{Atuação em comitês}

Sim

Não

$\begin{array}{ll}3(60,00) & 2(40,00) \\ 23(62,16) & 14(37,84) \\ 24(50,00) & 24(50,00)\end{array}$

\begin{tabular}{ll}
\hline & \\
$25(59,52)$ & $17(40,48)$ \\
$23(51,11)$ & $22(48,89)$ \\
$2(50,00)$ & $2(50,00)$
\end{tabular}

$0,719^{\neq}$

$1,000^{f}$

$\begin{array}{ll}3(60,00) & 2(40,00) \\ 1(100,00) & 0(0,00) \\ 46(54,12) & 39(45,88)\end{array}$

$0,322^{\neq}$

$\begin{array}{ll}24(50,00) & 24(50,00) \\ 23(65,71) & 12(34,29) \\ 2(50,00) & 2(50,00)\end{array}$

$0,141^{*}$

$24(48,00) \quad 26(52,00)$

$26(63,41) \quad 15(36,59)$

Legenda: *Qui-quadrado de Pearson; ${ }^{\neq}$Teste exato de Fisher. Fonte: Autores.

Ao se analisar a relação entre estresse e qualidade de vida, os dados mostram que o estresse interfere na qualidade de vida dos docentes, com significância estatística, em todos os domínios do WHOQOL-bref (Tabela 5). 
Tabela 5 - Análise inferencial da relação entre estresse e os diferentes domínios do WHOQOL-bref. Brasil, 2021.

\begin{tabular}{|c|c|c|c|}
\hline \multirow[t]{2}{*}{ Domínio } & \multicolumn{3}{|l|}{ Estresse } \\
\hline & n $(\%)$ & n $(\%)$ & P-valor \\
\hline & Sim & Não & \\
\hline Físico & & & $0,006 *$ \\
\hline Com QV & $21(42,00)$ & $29(58,00)$ & \\
\hline Sem QV & $29(70,73)$ & $12(29,27)$ & \\
\hline Psicológico & & & $0,039 *$ \\
\hline Com QV & $26(46,43)$ & $30(53,57)$ & \\
\hline Sem QV & $24(68,57)$ & $11(31,43)$ & \\
\hline Relações sociais & & & $0,001 *$ \\
\hline Com QV & $19(38,78)$ & $30(61,22)$ & \\
\hline Sem QV & $31(73,81)$ & $11(26,19)$ & \\
\hline Ambiente & & & $0,000 *$ \\
\hline Com QV & $18(35,29)$ & $33(64,71)$ & \\
\hline Sem QV & $32(80,00)$ & $8(20,00)$ & \\
\hline Auto-avaliação & & & $0,001 *$ \\
\hline Com QV & $19(38,78)$ & $30(61,22)$ & \\
\hline Sem QV & $31(73,81)$ & $11(26,19)$ & \\
\hline Geral & & & $0,000 *$ \\
\hline Com QV & $15(33,33)$ & $30(66,67)$ & \\
\hline Sem QV & $35(76,09)$ & $11(23,11)$ & \\
\hline
\end{tabular}

Legenda: *Qui-quadrado de Pearson. QV - Qualidade de vida. Fonte: Autores.

\section{Discussão}

Primordialmente é importante destacar que os resultados demonstraram que a autoavaliação de QV dos professores pesquisados, o IGQV, foi de $61,14 \%$. Isto demonstra que, mesmo diante das mudanças ocorridas em suas atividades cotidianas com o advento da pandemia, a maioria considera sua QV boa, de acordo com escala da Organização Mundial da Saúde, que classifica zero (0) como a pior percepção de QV e cem (100) a melhor (OMS, 1998). Em Revisão Sistemática realizada por Araldi et al (2021), cujo objetivo foi mapear os artigos publicados sobre qualidade de vida de professores atuantes em cursos de Ensino Superior e em estudo de Dalvoglio, Lettnin e Baldissera (2015), que objetivou conhecer características e qualidades metodológicas das pesquisas sobre a avaliação da QV com professores brasileiros, a avaliação de QV também foi considerada 
boa. No entanto, acredita-se que embora o momento pandêmico tenha evidenciado problemas de ordem social e econômica o fato de se tratar de servidor público, com estabilidade na carreira podem contribuir para uma avaliação positiva da QV pelos docentes.

Entre os participantes houve prevalência masculina, com idade entre 26 e 41 anos, casados/com companheiras(os) e com filhos. Este perfil difere da pesquisa de Souza et al. (2021), quando pesquisaram 588 professores brasileiros de instituições públicas estaduais e federais atuantes nos ensinos médio, técnico e superior, cujo objetivo foi identificar as condições, expectativas e capacidades de professores de cursos presenciais para a realização de atividades de ensino (letivas) remotas, mediadas por tecnologias digitais, em virtude do distanciamento social associado à pandemia da COVID-19, na qual houve predominância de mulheres, com idade variando entre 24 e 69 anos. A prevalência de docentes até 41 anos pode ser justificada pelo tempo de existência da instituição pesquisada, que é de 12 anos apenas.

Ao analisar-se este perfil sob a ótica dos domínios relacionados à QV, observa-se que a situação conjugal interferiu positivamente no domínio "Físico", que diz respeito à "dor e desconforto, energia e fadiga, sono e repouso, mobilidade, atividades da vida cotidiana, dependência de medicação ou de tratamentos e capacidade de trabalho" (Gordia, Quadros, Oliveira \& Campos, 2011); e que foi o domínio com maior influência negativa na QV dos docentes. Sob esta ótica pode-se inferenciar que a presença de um companheiro(a), traz à tona a associação do conforto físico às relações conjugais, que podem denotar a existência de atividades de lazer no cotidiano do casal, bem como, a realização de atividades físicas. Esta situação conjugal, apesar de não apresentar relação estatisticamente significativa, também impactou positivamente no domínio das "Relações sociais", que foi um dos domínios com melhor impacto na QV.

Associando-se a isto e ainda sobre o perfil, o maior quantitativo de docentes investigados foram da área das Ciências humanas e sociais aplicadas, seguido pela área das Ciências da saúde, que atuam no ensino técnico subsequente ou na graduação/tecnólogo, em regime de dedicação exclusiva para Instituição o que implica no impedimento do exercício de outra atividade remunerada, pública ou privada, com as exceções previstas em lei (Brasil, 2012). Concorda-se com de Souza (2021) quando coloca que esta realidade caracteriza uma dimensão do ensino na qual o docente consegue organizar-se de maneira tal a atender as demandas advindas do ensino, pesquisa e extensão com qualidade.

Por outro lado, com a necessidade de trabalhar aulas online, provenientes do ensino remoto, observou-se que a maioria dos docentes ministra grande carga horária de atividades remotas de ensino, sendo que 15 dos participantes relatam o sentimento de sobrecarga de trabalho. Evidencia-se que cargas horárias de aulas excessivas remetem ao aumento de demanda no preparo destas aulas, o que se soma ao tempo dispensado para ministrá-las. Adicionalmente a esta sobrecarga, o docente precisou empregar e utilizar novas ferramentas de ensino, pelo menos a maioria, com vistas a aprimorar a prática pedagógica neste novo contexto educacional em que o professor é mediador do conhecimento, e busca ampliar suas competências e habilidades para estimular a aprendizagem e a criatividade dos estudantes. No mais, destaca-se a necessidade de constante planejamento e reformulação das práticas pedagógicas, fato este que contribui para o grande montante de tempo a ser dispensado à sua atividade profissional (Rodrigues, Araújo, Silva, Dias \& Carvalho, 2021).

Em estudo realizado com servidores de uma instituição de ensino básico, técnico e tecnológico, os docentes relatam que as atividades de forma remota ampliaram a carga horária diária de dedicação ao trabalho, seja pela necessidade de aprender a utilizar recursos tecnológicos, de conciliar a vida pessoal e profissional, a dificuldade de concentração e de organização do tempo neste novo modo de vida e, ainda, a falta de estrutura doméstica para o trabalho (Frainer, Braz, Migliorini, Rosa, Silva et al, 2020). Estudo de Souza et al. (2021) reforça estes achados ao demonstrar que o trabalho realizado no ambiente domiciliar está relacionado à desmotivação e a baixa produtividade. Entende-se que todo este contexto contribui para os elevados índices de estresse apresentados pelos docentes nesta pesquisa.

Observa-se, de forma complementar, que além da carga horária de ensino elevada; na variável "atuação em comitês", 
15\% dos docentes que possuem esta carga horária adicional possuem menor QV, apesar do valor de p não ser significativo, pois esta condição justifica a influência negativa no domínio "Físico” da QV destes docentes. Estudo de Araújo et al. (2019), realizado com a finalidade de avaliar a qualidade de vida no trabalho (QVT) de docentes universitários encontrou perfil formativo e laborativo semelhantes ao desta pesquisa: maioria de docentes com titulação de doutor; que exercem suas atividades em regime de 40 horas semanais com dedicação exclusiva; com disponibilidade de carga horária para atividades extraclasse e burocráticas, bem como para atividades extras dentro da instituição. Apesar do estudo ter sido conduzido somente com docentes do ensino superior, o perfil se assemelha ao encontrado nesta pesquisa, com especial destaque para as atividades extras desempenhadas pelos docentes, que também foram apontadas como fator de piora da QV.

No mais, as contribuições negativas identificadas pelas facetas "dependência de medicamentos ou de tratamentos" e "dor e desconforto" ratificam esta perspectiva; diante da realidade de trabalho imposta pela pandemia, os docentes passaram a permanecer muito tempo em frente à computadores, o que além de comprometer a saúde física, com o aparecimento de lesões osteoarticulares e de visão (relacionados ao trabalho repetitivo, iluminação e postura inadequadas), podem ter influenciado na diminuição da realização de atividade física; além da redução já ocasionada pelo isolamento social. Estes dados são corroborados por Taylor (2019) que evidencia que o ensino, associado às atividades administrativas, é capaz de alterar a dinâmica cotidiana do docente, sobrecarregando-o e consequentemente trazendo sintomas físicos.

Por outro lado, a atuação em comitês institucionais demonstrou influenciar positivamente a QV no que diz respeito ao domínio das "Relações Sociais", visto que nesse período de contato e convivência com seus pares, há a possibilidade de troca, de divisão de suas angústias, de descontração, entre outros; ou seja, pode ser um dos momentos de desvio do "foco" pelo docente.

A maioria dos participantes possui titulação de doutor e o fato que chama a atenção é que este foi um fator significativo para melhoria da QV. A instituição pesquisada incentiva a qualificação profissional, no nível stricto sensu, e oferece um plano de carreira com a mudança de nível conforme a capacitação docente com aporte no padrão de vencimento. É possível considerar que uma melhor retribuição, aliada a estabilidade empregatícia advinda do concurso público, confiram segurança financeira e proporcionem melhores condições de vida de maneira geral. Isto pode ser reforçado pelos achados de que o "transporte" e o "ambiente do lar" foram duas das facetas mais bem avaliadas, uma vez que a condição financeira possibilita ao indivíduo que seu deslocamento seja feito com veículo próprio, bem como melhores condições habitacionais. Pode-se inferir que esta condição também contribuiu para uma avaliação positiva da faceta "autoestima", que pode estar relacionada à própria titulação e/ou condições sociais e financeiras nas quais este se encontra.

Ao avaliar-se a contribuição dos domínios para QV, observou-se que o domínio "Meio ambiente" contribui positivamente, seguido das "Relações sociais" e "Psicológico", enquanto o domínio "Físico" teve menor contribuição para o IGQV. Estes resultados se assemelham aos achados de Araldi, Poulsen, Guimarães \& Farias (2021) e diferem dos resultados encontrados por Dalvoglio, Lettnin \& Baldissera (2015), que evidenciaram o domínio "Meio ambiente" como o que menos contribuiu com a QV dos pesquisados. Importante ressaltar que este domínio está relacionado às condições de segurança física e proteção (WHO, 2005), o que vem ao encontro com a situação pandêmica, pois os docentes estão desenvolvendo suas atividades profissionais no ambiente domiciliar, o que lhes garante o conforto e acolhimento necessários às condições impostas pela pandemia, fato este reforçado pela faceta "ambiente do lar", que é uma das facetas que influencia positivamente a QV dos docentes.

Observou-se que os docentes que têm filhos tiveram uma melhora significativa na QV em seu domínio "Psicológico". Destaca-se que a maioria dos docentes tem um único filho. Este dado contradiz outros estudos, como o de Araújo, Fé, Araújo, Oliveira, Moura \& Silva (2019), que demonstrou que não ter filhos resulta em melhor qualidade de vida no trabalho de docentes, e que quanto mais filhos menor a qualidade de vida. Este resultado chama a atenção, em especial neste momento de 
sobrecarga vivenciada pelos docentes, no qual as atividades laborativas e de cuidado com a família e com o lar se sobrepuseram. Em estudo de Frainer et al. (2020), 50\% dos entrevistados relataram muitas dificuldades para conciliar as atividades domésticas e de trabalho, em especial os que têm filhos, uma vez que estes também se encontram em atividades remotas e necessitam de auxílio para desenvolver suas tarefas.

Outro dado que chama atenção é em relação aos docentes que possuem algum tipo de deficiência. Mesmo não havendo comprovação estatística de sua influência na QV, $80 \%$ dos entrevistados com deficiência afirmaram ter boa qualidade de vida. Analisando-se estudos realizados, envolvendo trabalho e deficiência, percebe-se que o perfil comumente encontrado, de pessoas com deficiência, inseridas no mercado de trabalho é de jovens, sem ensino superior e com baixa renda (Pereira \& Passerino, 2012; Vasconcelos, 2010; Baptista \& Silva, 2010), aspectos que divergem do encontrado na presente pesquisa. Coutinho, França, Coura, Medeiros \& Aragão (2017) indica que a QVT de pessoas com deficiência tende a ser influenciada por fatores como remuneração mensal, carga horária de trabalho e quantidade de trabalho adequada à condição da pessoa. Infere-se que tais fatores se encontram bem ajustados, contribuindo para o alcance de boa QV entre as pessoas com deficiência participantes da pesquisa. Neves-Silva, Prais e Silveira (2015) estabelecem uma correlação entre deficiência e ambiente de trabalho na qual quanto mais acessível, acolhedor e seguro for esse espaço, mais impactará positivamente na promoção da saúde deste segmento populacional.

O estresse interfere na QV dos docentes, em todos os domínios, o que denota o momento pelo qual os participantes estão passando. Associado a todas as angústias advindas de uma pandemia, causada por um vírus, que tem elevado os índices do número de casos e de letalidade dia após dia. Há a experiência de atividades remotas, que Santos (2020) considera um fator predisponente à desmotivação, negativismo e esgotamento, o que pode interferir diretamente nas situações de estresse. Saraiva, Traversini e Lockmanck (2020) acrescentam ainda que nem sempre os docentes são capazes de perceber este contexto.

Estes achados assemelham-se ao estudo de Barbosa, Silva e Quaresma (2020), que pesquisaram o nível de estresse e atividade física em docentes de um Instituto Federal da região norte do Brasil e identificaram que os participantes apresentaram um elevado nível de estresse, devido à inúmeras condições, tais como: elevada carga horária de trabalho, atividades administrativas e demandas relacionadas à produções científicas. Outro achado importante deste estudo foi ratificado por esta mesma pesquisa, pois Barbosa, Silva Peixoto, Quaresma \& Maciel (2020) evidenciaram que a prática de atividade física insuficiente contribui diretamente para o aumento do estresse entre os docentes.

Nesta pesquisa houve predomínio de docentes com estresse em fase de resistência. Esta fase é classificada como o segundo estágio do estresse, compreendido entre quatro estágios consecutivos: alerta, resistência, quase exaustão e exaustão (Lipp, 2015). Tal classificação tem como cerne a capacidade fisiológica dos indivíduos adaptarem-se aos eventos estressores, seria a Síndrome da Adaptação Geral ao Estresse (Pagliarone e Sforcin, 2009).

A segunda fase do estresse divide-se em resistência e quase exaustão. A resistência ocorre quando o agente estressor é mantido e o indivíduo não consegue restabelecer a homeostasia do corpo. Ocorre redução da produtividade e sintomas como mudanças de humor, insônia, gastrite, irritabilidade e hipertensão são comuns. A quase exaustão é quando a resistência psicológica não consegue suportar o estresse da fase anterior e os sintomas se intensificam, levando o indivíduo a experienciar momentos de ansiedade e desconforto frequentes podendo evoluir para infartos, úlceras, psoríase, depressão entre outros comprometimentos de vários órgãos (Lipp, 2015).

Desta forma, os docentes que se encontram nesta fase de resistência podem apresentar comprometimentos relacionados ao desempenho profissional e QV, pois é neste momento que estão presentes manifestações de desgaste físico e mental, bem como, sucessivas tentativas de reequilíbrio do organismo (Lima et al., 2021). Ou seja, neste momento há uma busca de adaptação do organismo a estes fatores que, caso não aconteça, o indivíduo passará para a próxima fase, que é a exaustão. 
É importante considerar que as condições de trabalho e sociais impostas pela pandemia vem se estendendo por mais de um ano na vida dos docentes, o que caracteriza um fator de estresse adicional, que pode levar a piora do quadro apresentado, com a evolução do estresse para a fase de exaustão, comprometendo tanto a saúde física quanto psíquica dos docentes (Lipp, 2015). Jacomini e Penna (2016) ponderam que o estresse patológico compromete, além da saúde dos docentes, seu exercício profissional e a qualidade da educação, fatos estes que precisam ser levados em consideração no planejamento das instituições de ensino.

Quanto aos sintomas predominantes, a maioria dos docentes com estresse relataram ter sintomatologia psicológica. Infere-se que este achado pode ter relação com a faceta "pensamentos negativos", que foi uma das que mais contribuiu para redução da QV dos participantes. Esta variável pertence ao domínio "Psicológico", que teve relação significativa com a ocorrência de estresse. O predomínio de sintomas psicológicos deve ser levado em consideração para formulação de estratégias de enfrentamento ao estresse, pois caracterizam que este é o ponto com maior vulnerabilidade no grupo pesquisado (Lipp, 2015).

Destaca-se que nenhuma das variáveis sociodemográficas pesquisadas teve relação significativa com o estresse, contudo os homens apresentaram-se mais estressados que as mulheres. Frainer et al (2020) apontam que isto pode ser devido ao fato de as mulheres estarem mais acostumadas às atividades domésticas e a conciliá-las com as atividades de trabalho, devido a questões históricas e culturais, fato que favorece a sua adaptabilidade.

Entre as limitações desta pesquisa é possível citar a amostragem por conveniência, em que a chance de viés de seleção é maior que na amostragem probabilística. Entretanto, esta pesquisa teve potencialidades: o fato de ser uma pesquisa original é um grande diferencial em tempos de pandemia, com inúmeras restrições; a abordagem é inovadora em tempos de COVID-19, capaz de gerar hipóteses sobre o estresse e qualidade de vida dos docentes de uma instituição de ensino pública que envolve diferentes modalidades de ensino.

\section{Conclusão}

Os dados demonstram que a maioria dos docentes pesquisados se apresentam com estresse, em sua fase intermediária e com predominância de sintomas psicológicos associados. Os homens mostraram-se com maior incidência de estresse, apesar de não haver associação estatística significativa. No tocante a qualidade de vida, a situação conjugal, presença de filhos, titulação e participar de comitês institucionais tiveram impacto estatisticamente significativo. No mais, o estresse mostrou-se um fator que reduziu significativamente a qualidade de vida em todos os seus domínios. Estes achados denotaram uma construção complexa, na qual interagem aspectos individuais, subjetivos e multidimensionais.

Avaliar os fatores que contribuem para melhor qualidade de vida e redução do estresse, bem como os que levam a redução da qualidade de vida e aumentam o estresse, são essenciais para se programar estratégias que favoreçam a saúde mental dos docentes neste momento de pandemia. Os resultados desta pesquisa reforçam a importância de se promover ações que objetivem a redução dos níveis de estresse, os quais se mostraram elevados e já em fase de resistência, impactando diretamente na qualidade de vida.

É primordial que as instituições de ensino busquem estratégias coletivas de promoção à saúde, em especial a mental, com vistas a reduzir o impacto da pandemia para os docentes. Como demonstrado pelos resultados, incentivar a participação dos docentes em atividades como comitês institucionais pode favorecer o domínio das relações sociais e ser um fator de melhora da qualidade de vida, em especial neste momento em que o distanciamento social se faz necessário. No mais, estratégias que visem a redução da sobrecarga intelectual, física e social dos docentes são importantes, bem como a promoção de espaços para compartilhar medos, angústias e outros sentimentos.

Sugere-se a realização de estudos com docentes que vivenciam outras realidades de ensino, bem como com os 
discentes de diferentes níveis de ensino. Conhecer distintas realidades contribui para o planejamento de estratégias mais eficazes e que englobe todos os atores envolvidos no processo, o que pode gerar melhores resultados.

\section{Referências}

Alvarenga, R., Martins, G. C., Dipe, E. L., Campos, M. V. de A., Passos, R. P., Lima, B. N., ... \& Fileni, C. H. P. (2020). Percepção da qualidade de vida de professores das redes públicas e privadas frente à pandemia do covid-19. Revista CPAQV-Centro de Pesquisas Avançadas em Qualidade de Vida, 12(3), 2. http://www.cpaqv.org/revista/CPAQV/ojs-2.3.7/index.php?journal=CPAQV\&page=article\&op=view\&path[]=538

Araldi, F. M., Poulsen, F. F., Guimarães, A. C. de A., \& Farias, G. O. (2021). Qualidade de vida de professores do ensino superior: uma revisão sistemática Revista

Retos,

459-470.

https://www.researchgate.net/publication/349072047_Qualidade_de_vida_de_professores_do_ensino_superior_uma_revisao_sistematica_Quality_of_life_of_ higher_education_teachers_a_systematic_review doi: 10.47197/retos.v0i41.82136

Araújo, A. L de.; Fé, E. de M.; Araújo, D. A. de M.; Oliveira, E. S. de; Moura, I. H. de. \& Silva, A. R. V. (2019). Avaliação da Qualidade de Vida no Trabalho de Docentes Universitários: Revista de Enfermagem do Centro Oeste Mineiro, 9, e 3195. http://www.seer.ufsj.edu.br/index.php/recom/article/view/3195 doi: https://doi.org/10.19175/recom.v9i0.3195

Avelino, W., \& Mendes, J. (2020). A realidade da educação brasileira a partir da COVID-19 Boletim de Conjuntura (BOCA), 2(5), 56-62. https://revista.ufrr.br/boca/article/view/AvelinoMendes/2892. doi:http://dx.doi.org/10.5281/zenodo.3759679

Barbosa, T. C., Silva, A. P., Peixoto, F.R., Quaresma, F. R. P., \& Maciel, E. S. (2020) Estresse percebido e nível de atividade física em docentes de um Instituto Federal. Revista Saúde em Revista 20(52) 47-56. https://www.metodista.br/revistas/revistas-unimep/index.php/sr/article/view/4568

Brasil. Ministério da Educação. Gabinete do Ministro. (2020a). Ministério da Saúde. PORTARIA nº 188 de 3 de fevereiro de 2020 . Declara emergência de saúde pública de importância nacional (ESPIN) em decorrência da Infecção Humana pelo novo Coronavírus (2019-nCoV). Diário Oficial da União, Brasília. https://www.in.gov.br/en/web/dou/-/portaria-n-188-de-3-de-fevereiro-de-2020-241408388

Brasil. Ministério da Educação. Gabinete do Ministro. (2020b). PORTARIA n ${ }^{\circ} 544$ de 16 de junho de 2020. Dispõe sobre a substituição das aulas presenciais por aulas em meios digitais, enquanto durar a situação de pandemia do novo coronavírus - Covid-19. Diário Oficial da União, Brasília. https://abmes.org.br/arquivos/legislacoes/Portaria-mec-544-2020-06-16.pdf

Brasil. Ministério da Educação. Gabinete do Ministro. (2012)., LEI no 12.772/2012 de 28/12/2012... Previsão Legal nos art. 20 a 26. https://www.planalto.gov.br/ccivil_03/_ato2011-2014/2012/lei/112772.htm

Baptista, A. K., \& Silva, F. C. M. (2010) Perfil demográfico e do emprego das pessoas com deficiência no município de Belo Horizonte, Minas Gerais Brasil. Revista Médica de Minas Gerais, Belo Horizonte, 20(1), 37-48. http://www.rmmg.org/artigo/detalhes/172

Coutinho, B. G., França, I. S. X .de., Coura, A. S., Medeiros, K. K. A. S., \& Aragão, J. S. (2017). Qualidade de Vida no Trabalho de pessoas com deficiência física. Trabalho, Educação e Saúde, 15(2), 561-573. https://www.scielo.br/scielo.php?script=sci_arttext\&pid=S1981-77462017000200561\&lng=en\&nrm=iso doi: https://doi.org/10.1590/1981-7746-sol00061 doi:https://doi.org/10.1590/1981-7746-sol00061

Davoglio, T. R., Lettnin, C. da C., \& Baldissera, C. G. (2015). Avaliação da qualidade de vida em docentes brasileiros: uma revisão sistemática. Revista ProPosições, 26(3), 145-166. Disponível em: https://www.scielo.br/pdf/pp/v26n3/0103-7307-pp-26-03-0145.pdf doi:_https://doi.org/10.1590/01037307201507807

Jiang, F., Deng, L., Zhang, L., Cai, Y., Cheung, C. W., \& Xia, Z. (2020). Review of the Clinical Characteristics of Coronavirus Disease 2019 (COVID-19). Journal of general internal medicine, 35(5), 1545-1549. doi: 10.1007/s11606-020-05762-w

Fleck, M. P., Louzada, S., Xavier, M., Chachamovich, E., Vieira, G., Santos, L., \& Pinzon, V. (2000). Aplicação da versão em português do instrumento abreviado de avaliação da qualidade de vida "WHOQOL-bref. Revista de Saúde Pública, 34(2), 178-183. doi: https://doi.org/10.1590/s003489102000000200012 .

Frainer, D. E. S.; Braz, F. J.; Migliorini, G.; Rosa, H. I.; Silva, J. V. B. M.; Leal, M. ...\& Fruneaux, S. C. (2020). Trabalho remoto: um olhar dos servidores e servidoras do Instituto Federal Catarinense. SINASEFE Litoral, 1(3). https://www.potemkin.sinasefe-ifc.org/wp-content/uploads/2020/12/potemkin3-04Trabalho-remoto-um-olhar-dos-servidores-e-servidoras.pdf

Garcia, L. P., \& Duarte, E. (2020) Nonpharmaceutical interventions for tackling the COVID-19 epidemic in Brazil. Epidemiologia e Serviços de Saúde 29(2) e 2020222. scielo.br/scielo.php?script=sci_arttext\&pid=S2237-96222020000200100\&lng=en\&nrm=iso\&tlng=en doi:https://doi.org/10.5123/s1679. 49742020000200009

Gordia, A. P., Quadros, T. M. B., Oliveira, M. T. C., \& Campos, W. (2011). Qualidade de vida: contexto histórico, definição, avaliação e fatores associados. Revista brasileira de qualidade de vida 3(1), 40-52. https://periodicos.utfpr.edu.br/rbqv/article/view/812/625 doi: 10.3895/S2175-08582011000100005.

Holmes , E.A., O'Connor R. C., Perry. H., Tracey. I, Wessely, S., Arseneault. L. ...\& Bullmore, E. (2020). Multidisciplinary research priorities for the COVID19 pandemic: a call for aHction for mental health science. Disponível em: https://www.thelancet.com/journals/lanpsy/article/PIIS2215-0366(20)301681/fulltext doi: https://doi.org/10.1016/S2215-0366(20)30168-1. Acesso em: 24 mar. 2021

Hulley, S. B. Delineando a pesquisa clínica: uma abordagem epidemiológica / Stepehn B. Hulley et al.; tradução Michel Schimidt Duncan. - $4^{a}$ ed. - Porto Alegre: Artmed, 2015. 384 p.

Jacomini, M. A.; \& Penna, M. G. D. O. (2016) Carreira docente e valorização do magistério: condições de trabalho e desenvolvimento profissional. ProPosições, 27(2), 177-202. https://periodicos.sbu.unicamp.br/ojs/index.php/proposic/article/view/8647238 doi: https://doi.org/10.1590/1980-6248-2015-0022. 
Lima, M. M. M., M'Batna, A. J., Felício, J. F., Ferreira, D. Silva, Dutra, F. C da S., Cavalcanti, M. C.e S. L., Santos, L.V.F ., Monte, A. S., Cavalcante, C.M., \& Amaral, J. F do (2021). Perfil de manifestação de estresse em universitários: um estudo transversal. Revista Enfermagem Atual In Derme, 95(33). revistaenfermagematual.com/index.php/revista/article/view/903 doi: https://doi.org/10.31011/reaid-2021-v.95-n.33-art.903

Lipp, M. N. Manual do Inventário de Sintomas de Stress para Adultos de Lipp (ISSL). 3ed. Casa do Psicólogo, 2015.

Nhantumbo, T. L. (2020) Capacidade de resposta das instituições educacionais no processo de ensino-aprendizagem face à pandemia de COVID-19: impasses e desafios. Revista EDUCAmazônia - Educação Sociedade e Meio Ambiente, Humaitá, (2), 556-571 https://periodicos.ufam.edu.br/index.php/educamazonia/article/view/7851/5535.

Neves-Silva, P.; Prais, F. G., \& Silveira, A. M. Inclusão da pessoa com deficiência no mercado de trabalho em Belo Horizonte, Brasil: cenário e perspectiva. Revista Ciência \& Saúde Coletiva, 20(8), 2549-2558. https://www.scielo.br/scielo.php?script=sci_arttext\&pid=S1413-81232015000802549 doi: https://doi.org/10.1590/1413-81232015208.17802014.

Organização Mundial de Saúde (OMS). Promoción de la salud: Glosario. Ginebra: Organización Mundial de la Salud; 1998. https://apps.who.int/iris/bitstream/handle/10665/67246/WHO_HPR_HEP_98.1_spa.pdf?sequence=1\&isAllowed=y

Ornell, F., Schuch, J. B., Sordi, A. O., \& Kessler, F. H. P. (2020). "Medo pandêmico" e COVID-19: carga e estratégias de saúde mental. Revista Brasileira de Psiquiatria $\quad, \quad 42 \quad(3), \quad 232-235 . \quad$ https://www.scielo.br/scielo.php?script=sci_arttext\&pid=S1516-44462020000300232\&tlng=en doi: https://doi.org/10.1590/1516-4446-2020-0008

Pagliarone, A. C., \& Sforcin, J. N. (2009) Estresse: revisão sobre seus efeitos no sistema imunológico. Revista Biosaúde 11(1), 57-90. http://www.uel.br/revistas/uel/index.php/biosaude/article/view/24304/17878.7.

Pedroso, B.; Pilatti, L. A.; \& Reis, D. R. (2009) Cálculo dos escores e estatística descritiva do WHOQOL-100 utilizando o Microsoft Excel. Revista Brasileira de Qualidade de Vida. https://periodicos.utfpr.edu.br/rbqv/article/view/366 1(1), 23-32 doi: 10.3895/S2175-08582009000100003

Pereira, A. C. C. \& Passerino, L. (2012) Um estudo sobre o perfil dos empregados com deficiência em uma organização. Revista Brasileira de Educação Especial, $\quad 18(2), \quad 245-264 . \quad$ https://www.scielo.br/scielo.php?pid=S1413-65382012000200006\&script=sci_abstract\&tlng=pt doi: https://dx.doi.org/10.1590/S1413-65382012000200006.

Pinto, K. S.; Dias, A. R.; Salera, I. B.; Silva, J. B. F. \& Marinho, V. L. (2021) Quality of life in university professors: astudy at a university in southern Tocantins. Retrieved from:Amazônia Science \& Health 9(1), 14-24. http://ojs.unirg.edu.br/index.php/2/article/view/3307

Rodrigues, C. F. A.; Araújo, H. R.; Silva, M. V.; Dias, T. M. da S. \& Carvalho, E. T. (2021). Os desafios das práticas docentes em meio a situação enfrentada no ensino a distância. Brazilian Journal of Development, 7(1), 3520-3527. https://editorarealize.com.br/artigo/visualizar/69214

Rodrigues, N. P. A., Silva, D. R., Garcia Jr, E.A., Silva Jr., E. F., Gomes, R. S., Fernandes, K. F. D., \& Oliveira, K. Á. R. (2021). Disclosure of information on hygiene and changing habits during the Covid-19 pandemic. Research, Society and Development, 10(1), e 30910111739.https://www.rsdjournal.org/index.php/rsd/article/view/11739 doi: https://doi.org/10.33448/rsd-v10i1.11739 .

Saraiva, K., Traversin, C., \& Lockmannk, K. (2020). Educação em tempos de COVID-19: ensino remoto e exaustão docente. Revista Prax Educativa. (15) 124. https://revistas2.uepg.br/index.php/praxiseducativa/article/view/16289 doi: https://doi.org/10.5212/PraxEduc.v.15.16289.094

Santos, H. (2020) Os desafios de educar através da Zoom em contexto de pandemia: investigando as experiências e perspectivas dos docentes portugueses. Revista Praxis Educativa $\quad$ 1-17 https://www.researchgate.net/publication/343451836_Os_desafios_de_educar_atraves_da_Zoom_em_contexto_de_pandemia_investigando_as_ex periencias_e _perspetivas_dos_docentes_portugueses doi: 10.5212/PraxEduc.v.15.15805.091

Souza, A. S. R., Amorim, M. M. R., Melo, A. S. O. de., Delgado, A. M., Florêncio, A. C. M. C. C., Oliveira, T. V., Lira, L.C.S., ... \& Katz, L. (2021). Aspectos gerais da pandemia de COVID-19. Revista Brasileira de Saúde Materno Infantil, 21(Suppl.1), 29-45. https://www.scielo.br/scielo.php?script=sci_arttext\&pid=S1519-38292021000100029\&lng=en\&nrm=iso\&tlng=pt doi: https://dx.doi.org/10.1590/18069304202100 s 100003

Souza, G. H. S., Jardim, W. S., Marques, Y. B., Lopes Jr., G., Santos, A. P. S. dos, \& Liberato, L. de P. (2021). Emergency Remote Education (ERE): An empirical study on Educational Capabilities and Teaching Expectations during the COVID-19 Pandemic. Retrieved from: Research, Society and Development, 10(1), e37510111904. https://rsdjournal.org/index.php/rsd/article/view/11904 doi: https://doi.org/10.33448/rsd-v10i1.11904

Taylor, S. (2019). The psychology of pandemics: Preparing for the next global outbreak of infectious disease. Newcastle upon Tyne: Cambridge Scholars Publishing.

Organização Mundial de Saúde (OMS). Promoción de la salud: Glosario. Ginebra: Organización Mundial de la Salud; 1998. https://apps.who.int/iris/bitstream/handle/10665/67246/WHO_HPR_HEP_98.1_spa.pdf?sequence=1\&isAllowed=y

Vasconcelos, F. D. (2010) O trabalhador com deficiência e as práticas de inclusão no mercado de trabalho de Salvador, Bahia. Revista Brasileira de Saúde Ocupacional, $\quad 35(121), 41-52 . \quad$ https://www.scielo.br/scielo.php?pid=S0303$76572010000100006 \&$ script=sci_abstract\&tlng=pt\#: :text=O\%20autor\%20analisou\%20pol\%C3\%ADticas\%20e,de \%20trabalho\%20em\%20Salvador\%2C\%2 0Bahia.\&text=O\%20autor\%20constatou\%20que\%20o,trabalho\%20das\%20pessoas\%20com\%20defici\%C3\%AAncia doi: https://doi.org/10.1590/S030376572010000100006

WHO. World Health Organization. Statement on the second meeting of the International Health Regulations (2005) Emergency Committee regarding the outbreak of novel coronavirus (2019-nCoV) https://www.who.int/news/item/30-01-2020-statement-on-the-second-meeting-of-the-international-healthregulations-(2005)-emergency-committee-regarding-the-outbreak-of-novel-coronavirus-(2019-ncov)

WHO.World Health Organization. Quality of life assessment an annotated bibliography. Geneva: World Health Organization, 1994. https://apps.who.int/iris/bitstream/handle/10665/61629/WHO_MNH_PSF_94.1.pdf?sequence=1\&isAllowed=y 